\title{
Differential Expression of HCV Core Protein from Two Different Quasispecies
}

\author{
Kyung Lee Yu, and Ji Chang You* \\ National Research Laboratory of Molecular Virology, Department of Pathology, School of Medicine, \\ The Catholic University of Korea, Seoul 137-701, Republic of Korea
}

(Received April 7, 2009; Revised April 14, 2009; Accepted April 16, 2009)

\begin{abstract}
Hepatitis C virus (HCV) has genetic diversity like most of RNA viruses. HCV major genotypes are classified into several subtypes which are further divided into quasispecies having, genetically different but closely related variants. The HCV core that is a nucleocapsid protein located at the amino terminus of the viral polyprotein is relatively a conserved protein among the HCV isolates and thus it has been one of plausible targets for anti-HCV drug development. However, different quasispecies of HCV core gene have also been found. In this study, we compared the expression level of core protein between two different quasispecies of HCV genotype 1b. Our data demonstrate that a little differences of amino acid sequence lead to substantial difference of expression level. It might be another important reason of different pathogenesis among HCV infected patients.
\end{abstract}

Keywords: Hepatitis C virus, Core protein, Quasispecies

\section{INTRODUCTION}

Hepatitis $\mathrm{C}$ virus (HCV) is a major cause of chronic liver disease and liver transplants and over the 170 million persons chronically are infected with it world widely. It is a enveloped virus with a $9.6 \mathrm{~kb}$ positive-sense single-stranded RNA genome encoding a single open reading frame (ORF), which is processed by cellular and viral proteases to produce at least 10 structural and nonstructural proteins. It has been known now that there are at least six major genotypes and a number of subtypes of HCV which are generated due to high error rates in the viral RNA replication, (Choo et al., 1991). Among the different genotypes, $1 \mathrm{~b}$ and $2 \mathrm{a}$ are the most prevalent in Korea (Lim et al., 1997).

The HCV core is a nucleocapsid protein of 191 amino acids in length located at the amino terminus end of the viral polyprotein. Despite of HCV genetic variability, the core protein is relatively a conserved protein among the HCV isolates known to date. The core is also known as a multifunctional protein affecting progress of liver disease, immune response of the infected host, transcription of sev-

*Corresponding author

Tel: +82-2-2258-7312 Fax: +82-2-2258-7790

E-mail: jiyou@catholic.ac.kr eral cellular and viral genes, and cell apoptosis and regulation of viral translation (Moriya et al., 1998; McLauchlan, 2000; Shimoike et al., 2006). Therefore, the core protein is believed to be one of possible targets for antiviral drug development.

Due to lack of proof-reading function in RNA-dependent RNA polymerase, however, replication of $\mathrm{HCV}$ leads to numerous quasispecies within an infected individual (Martell et al., 1992). It is known that quasispecies play an important role in the pathogenesis, immune escape and drug resistance (Korenaga et al., 2001). Previous reports demonstrated that the core protein in different quasispecies of genotype $1 \mathrm{~b}$ have been related to different outcome of pathogenesis (Yan et al., 2008).

In this study, we have investigated two types of core protein expression plasmids harboring different quasispecies core gene of genotype $1 \mathrm{~b}$ (Genebank accession number HCU01214) to compare their expression levels. Our results show interestingly that two genes show a very definite difference in its expression level despite of slight differences in their amino acid composition. The result provides useful information for understanding of the expression of HCV core protein in host cells. 


\section{MATERIALS AND METHODS}

\section{Construction of expression plasmids}

The core gene was amplified with the following oligonucleotides: 5'CCCAGATCTAGGAGGTTTAAAATAATGAG CACGAATCCT3' and 5'AAACTGCAGTTAAGCGGAAGCTGGGACGGTCAG3' using pTre2COE1E2 harboring 5'UTR, core, E1 and E2 (kindly provided by Dr. H.J. Myung at the Hankuk University of Foreign Studies) or pCIN-neo core (kindly provided by K.L. Jang at the Pusan National University) having Type 1 and Type 2, respectively as a template. PCR products were inserted into pSE380 $\triangle$ laclq in which laclq gene was deleted from E.coli expression vector pSE380 (Invitrogen, USA) using the same restriction enzyme Bg/ll and Pstl. The PCR product of core Type 1 was blunted and inserted into pTre2 which was digested with Sacll and Xbal then blunted by the Klenow DNA polymerase (New England Biolabs, UK). The gag/pol region of pLP1 (Invitrogen, USA) was deleted by digestion with Smal and Pstl and replaced with core gene obtained from pSE380 $\triangle$ laclq/Type 1 and Type 2 which were digested with Dral and Pstl, respectively. All protein expression plasmids were engineered to have a termination codon (TAA) at the end of the protein coding sequence. All
A.

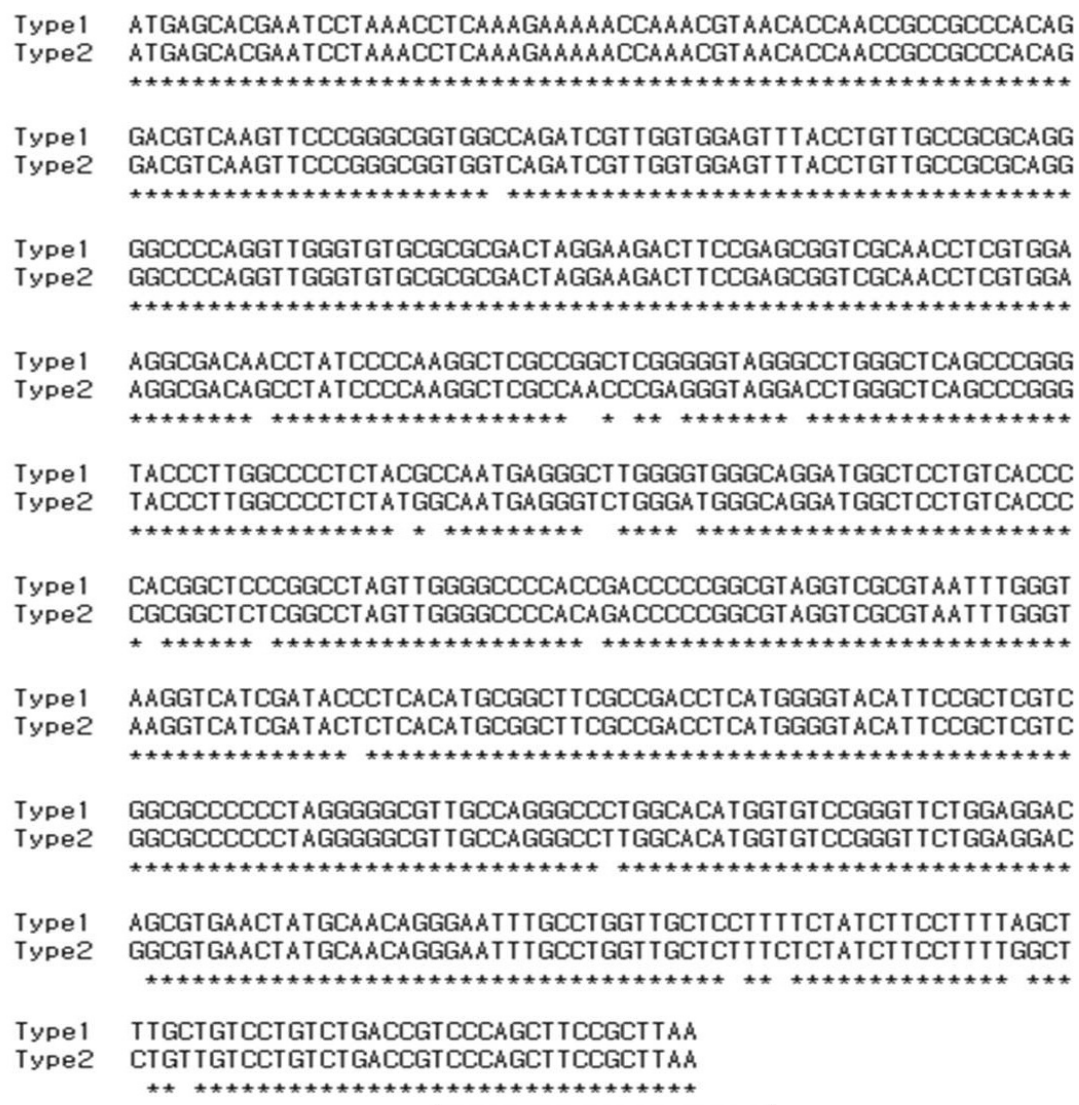

B. Type 1

Type2

Type 1

Type2

Type 1

Type2

Type 1 MSTNPKPQRKTKRNTNRRPQ OVKFPGGGQIVGGVVLLPRAGPRLGVRA TRKTSERSQPRG MSTNPKPQRKTKRN TNRRPQDVKFPGGGQI VGGVYLLPRA GPRLGURA TRKT SERSQPRG

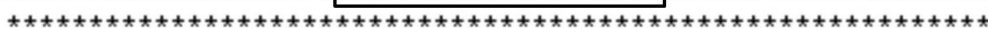

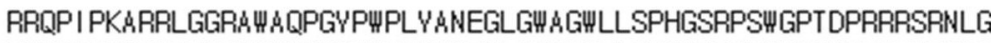
RRQPIPKARQPEGRT W \&QPGYPWPLYGNEGLGW\&GWLLSPRGSRPSWGPTDPRRRSRNLG

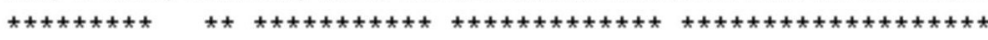

KVIDTLTCGFADLMGVIPLVGAPLGGVARALAHGVRVLEDSVNYATGNLPGCSFSIFLLA KVIDTLTCGF $A$ DLMGVIPLVGiPLGGVARALAHGVRVLEDGVNVATGNLPGCSFSIFLL

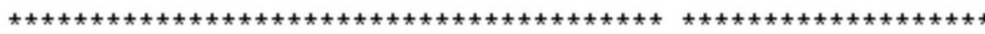

LLSCLTVPASA

LLSCLTVPASA
Fig. 1. Nucleotide sequence and amino acid sequence alignments of two different quasispecies of core used in this study. (A) Nucleotide sequence alignment. The asterisks indicate nucleotides matched. (B) Amino acid sequence alignment. The square mark indicates a recognition region of anti-core antibody used in this study. The asterisks indicate amino acid matched. 
cloned DNAs were confirmed by DNA sequencing

\section{The core protein expression in E.coli}

Core protein plasmids were transformed into $E$. coli strain JM109. Bacterial cells were grown at $37^{\circ} \mathrm{C}$ in LB medium containing $100 \mu \mathrm{g} / \mathrm{ml}$ of ampicillin until the culture reached an $\mathrm{OD}_{600}$ of 0.4 and induced by adding $1 \mathrm{mM}$ IPTG (Isopropyl- $\beta$-D-Thiogalactopyranoside Sigma Aldrich, USA). Incubation was continued for 4 hours after induction, and cells were harvested on every time points. An equal number of cells were harvested and centrifuged at 12,000 rpm for $1 \mathrm{~min}$. The pellet was resuspended with $80 \mu \mathrm{l}$ of sterile water and $20 \mu$ l of $5 \mathrm{X}$ sample buffer (60 mM Tris-HC, $2 \%$ SDS, $25 \%$ Glycerol, $0.1 \%$ bromophenol blue, $\beta$-mercaptoethanol).

\section{The core protein expression in mammalian cell}

293T cells were a maintained in Dulbecco's modified Eagle medium with $10 \%$ fetal bovine serum with penicillin and streptomycin (Invitrogen, USA) at $37^{\circ} \mathrm{C}$ in $5 \% \mathrm{CO}_{2}$. 293T cells were transfected with core protein expression plasmid using jetPEI (Polyplus-transfection, France). Cells were harvested 24 hours or 48 hours later and lysed with RIPA buffer (50 mM Tris- $\mathrm{HCl}, 150 \mathrm{mM} \mathrm{NaCl}, 1 \%$ nonident P-40, $0.5 \%$ deoxycholate, $10 \%$ SDS).

\section{SDS-PAGE and western blotting}

The cell lysate was boiled for $5 \mathrm{~min}$ and centrifuged for 5 $\min$ at $4^{\circ} \mathrm{C}$. Supernatants of each sample were separated by SDS-polyacryamide gel electrophoresis (SDS-PAGE) on a $12 \%$ acrylamid gel. Gel was stained by coomassie R250 staining solution $(0.05 \%$ coomassie brilliant blue R-250, 50\% methanol, $10 \%$ acetic acid) in case of E.coli lysates or was electrophoreticaly blotted onto a nitrocellulose filter membrane. The gel was then placed on the nitrocellulose membrane sandwiched between two pieces of $3 \mathrm{M}$ paper. The transfer apparatus was placed in transfer tank filled with $1 \mathrm{X}$ transfer buffer $(20 \mathrm{mM}$ Tris, $150 \mathrm{mM}$ Glycine, 20\% methanol, $0.038 \%$ SDS) and transferred for 1 hour at $300 \mathrm{~mA}$. The membrane was blocked with $20 \mathrm{ml}$ of $5 \%$ non-fat dry milk (BD, USA) in TBST (20 mM Tris$\mathrm{HCl}, 0.5 \mathrm{M} \mathrm{NaCl}, 0.05 \%$ Tween 20) for 1 hour with gentle shaking. Antibodies used were anti-core protein monoclonal antibody (Affinity BioReagents, USA) and anti- $\beta$-actin (Applied Biological Materials, Canada).

\section{RESULTS AND DISCUSSION}

In order to determine expression level of quasispecise core protein, we constructed two type core protein expression plasmids harboring different quasispecise of core gene of genotype $1 \mathrm{~b}$. Fig. 1 show differences in nucleotides and amino acid sequences between Type 1 and Type 2 of HCV core protein investigated in this study. There are total 23 nucleotide differences, which result in 16 synonymous codons and 7 amino acid changes.

To compare the expression level of these core proteins in bacterial and mammalian cells, we constructed two types of core expression plasmids for either in E.coli or mammalian cells as described in Materials and Methods, respectively. As shown Fig. 2, a high level expression of Type 1 core protein was observed in bacterial cells while that of Type 2 core protein was much less than Type 1.

In the case of mammalian cells, we employed initially pTre2-based pTre2/Type 1 and pCl-neo-based pCl-neo/ Type 2 eukaryotic expression plasmids. However, we were able to detect only a very low (Fig. 3A) or no expression (data not shown) for either forms of the proteins. Thus, we exploited another pLP1 based eukaryotic expression plasmids, which was further optimized in our laboratory for high level of protein expression in mammalian cell, resulting in pLP1/Type 1 and pLP1/Type 2. By using the expression vector, we were able to detect high level of Type 1 core protein as shown in Fig. 3. However, pLP1/Type 2 core still showed a very low expression level compared with pLP1/Type 1 (Fig. 3B), as was observed in E.coli. It was detected only when more exposure time was spent or cells were harvested at 72 hours after tranfection (data not shown). The transfection efficiency was estimated in the
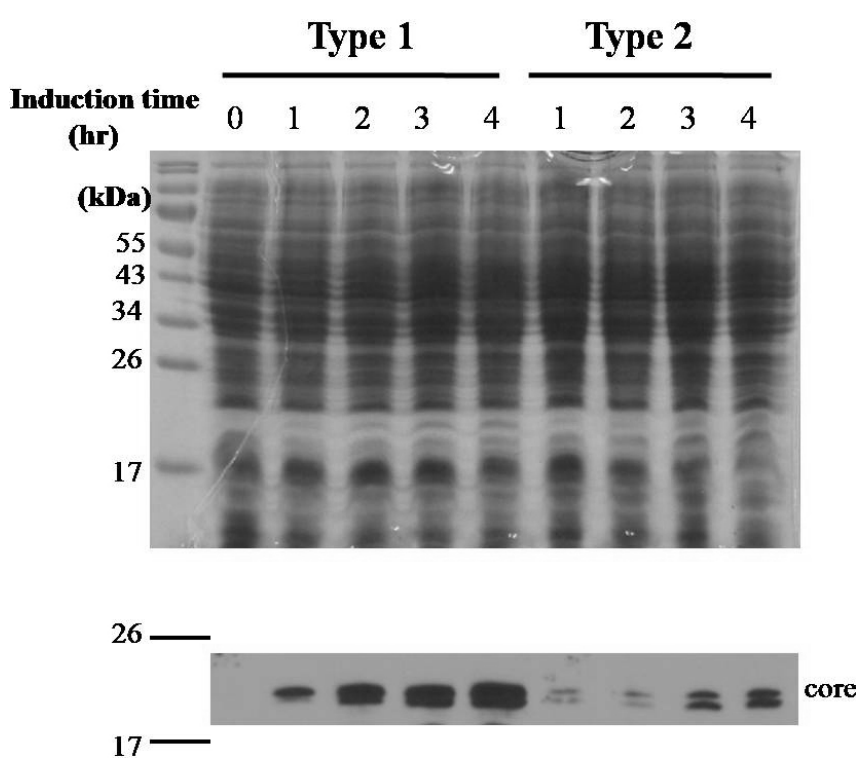

Fig. 2. Comparison of core protein expression level in E.coli. SDS-PAGE (top) shows the same volume of protein lysate used. The same volume of lysate was subjected to western blot analysis (bottom). 
A.

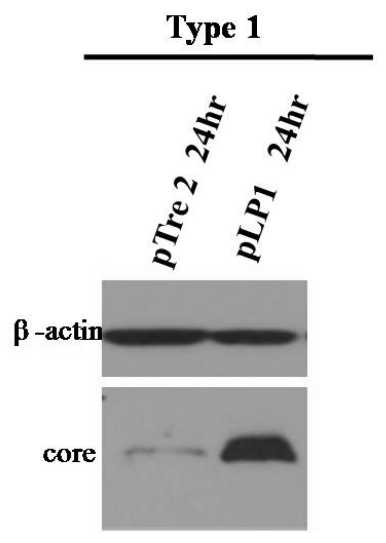

B.

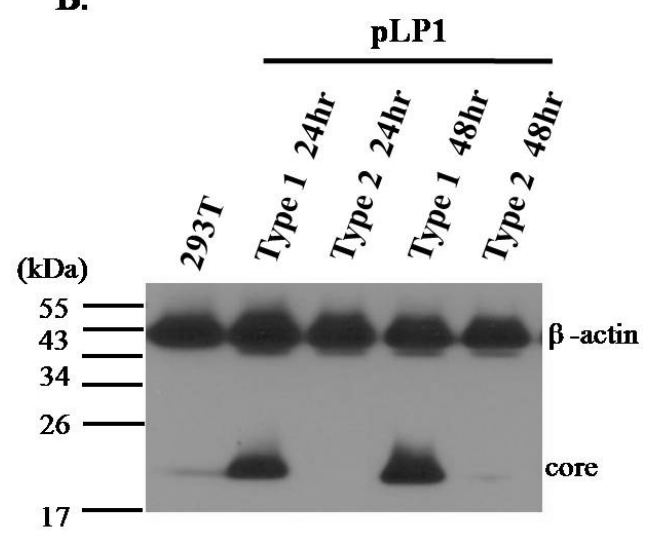

Fig. 3. Comparison of core protein expression level in mammalian cell. Each core protein expression plasmids is transfected into $293 \mathrm{~T}$ cell. (A) Different expression efficiency of Type 1 core using different expression plasmid system. (B) Comparison of core protein expression level between Type 1 and Type 2. Transfected cells were harvested at 24 hours and 48 hours and analysed as described in the materials and methods.
Table I. Different amino acids and rare codons between two different core genes

\begin{tabular}{ccc}
\hline Codon No. & Type 1 & Type 2 \\
\hline 70 & Arg CGG & Gln CAA \\
71 & Leu CTC & Pro CCC \\
72 & Gly GGG & Glu GAG \\
75 & Ala GCC & Thr ACC \\
87 & Ala GCC & Gly GGC \\
101 & His CAC & Arg CGC \\
110 & Thr ACC & Thr ACA \\
161 & Ser AGC & Gly GGC
\end{tabular}

Boldface indicates rare codon in E.coli.

similar range (\%) for both transfections based on expression of RFP vector that we use as an internal control for transfection efficiency. Of noted is that the anti-core monoclonal antibody used in this analysis recognizes an epitope ranging amino acids 21 to 40 region of the core protein in which no amino acid changes are observed (Fig. 1B). Therefore, no or very low level of detection of Type 2 core protein is not due to a problem of antibody used for detection.

When we analyzed codon usage and amino acid compositions for two types of core proteins, the followings are observed as shown in Table I. The amino acid changes in amino acid number 71,87 and 101 are conservative ones. On the other hand, changes in amino acid number 70,72 , 75 and 161 are all different property of amino acids; amino acid number 70 of Type 1 core is positively charged whereas that of Type 2 is uncharged, amino acid number 72 of Type 1 is uncharged while that of Type 2 is negatively charged, amino acid numbers 75 of Type 1 is nonpolar and hydrophobic while that of Type 2 is polar and hydrophilic and 161 of Type 1 are polar and hydrophilic ones whereas that of Type 2 are nonpolar and hydrophobic. We also noticed that Type 2 core encode more rare codons used in
E.coli than Type 1 as shown in boldface in Table I (Burns and Beacham, 1985). However, we did not observe much difference between Type 1 and 2 core proteins in their rare codon usage or overall codon frequency in mammalian cell (Carton et al., 2007).

Although it is not clear whether core protein directly affects on pathogenesis or whether it is outcome of host immune response. Previous report show that different quasispecies core proteins induced different level of cell apoptosis and proliferation inhibition (Yan and Mei et al., 2008). It appears that core protein related on the pathogenesis in some ways (Moriya et al., 1998).

Our data demonstrate clearly that different quasispecies of core gene have very different protein expression levels. Although the exact reason for the observed difference is not clear presently and awaits further analysis. But the results suggest that it could be a possible reason for the outcome of various pathogenesis between $\mathrm{HCV}$ infected individual.

\section{ACKNOWLEDGMENTS}

This work was supported by a National Research Laboratory grant (M10500000148-06J0000-14810), and a research grant (M10863000011-08N6300-01110) from the Korean Ministry of Science and Technology.

\section{REFERENCES}

Burns, D. M. and Beacham, I. R. (1985). Rare codons in E. coli and S. typhimurium signal sequences. FEBS Lett. 189, 318324.

Carton, J. M., Sauerwald, T., Hawley-Nelson, P., Morse, B., Peffer, N., Beck, H., Lu, J., Cotty, A., Amegadzie, B. and Sweet, R. (2007). Codon engineering for improved antibody expression in mammalian cells. Protein Expr. Purif. 55, 279-286. 
Choo, Q. L., Richman, K. H., Han, J. H., Berger, K., Lee, C., Dong, C., Gallegos, C., Coit, D., Medina-Selby, R., Barr, P. J., Weiner, A. J., Bradley, D. W., Kuo, G. and Houghton, M. (1991). Genetic organization and diversity of the hepatitis C virus. Proc. Natl. Acad. Sci. U. S. A. 88, 2451-2455.

Korenaga, M., Hino, K., Katoh, Y., Yamaguchi, Y., Okuda, M., Yoshioka, K. and Okita, K. (2001). A possible role of hypervariable region 1 quasispecies in escape of hepatitis $C$ virus particles from neutralization. J. Viral Hepat. 8, 331-340.

Lim, M. Y., Fry, K., Yun, A., Chong, S., Linnen, J., Fung, K. and Kim, J. P. (1997). Sequence variation and phylogenetic analysis of envelope glycoprotein of hepatitis G virus. J. Gen. Virol. 78(Pt 11), $2771-2777$.

Martell, M., Esteban, J. I., Quer, J., Genesca, J., Weiner, A., Esteban, R., Guardia, J. and Gomez, J. (1992). Hepatitis C virus $(\mathrm{HCV})$ circulates as a population of different but closely related genomes: quasispecies nature of $\mathrm{HCV}$ genome distribution. J. Virol. 66, 3225-3229.

McLauchlan, J. (2000). Properties of the hepatitis C virus core protein: a structural protein that modulates cellular processes J. Viral Hepatitis 7, 2-14.

Moriya, K., Fujie, H., Shintani, Y., Yotsuyanagi, H., Tsutsumi, T., Ishibashi, K., Matsuura, Y., Kimura, S., Miyamura, T. and Koike, K. (1998). The core protein of hepatitis $C$ virus induces hepatocellular carcinoma in transgenic mice. Nat. Med. 4 1065-1067.

Shimoike, T., Koyama, C., Murakami, K., Suzuki, R., Matsuura, Y., Miyamura, T. and Suzuki, T. (2006). Down-regulation of the internal ribosome entry site (IRES)-mediated translation of the hepatitis $C$ virus: critical role of binding of the stem-loop Illd domain of IRES and the viral core protein. Virology 345, 434-445.

Yan, X. B., Mei, L., Feng, X., Wan, M. R., Chen, Z., Pavio, N. and Brechot, C. (2008). Hepatitis C virus core proteins derived from different quasispecies of genotype $1 \mathrm{~b}$ inhibit the growth of Chang liver cells. World J. Gastroenterol. 14, 2877-2881. 